\title{
ON CERTAIN COMPARISON THEOREMS FOR SECOND ORDER LINEAR OSCILLATION
}

\author{
MAN KAM KWONG ${ }^{1}$
}

\begin{abstract}
It is shown that if $\left(p y^{\prime}\right)^{\prime}+q y=0$ on $[0, \infty)$ is oscillatory then $\left(p z^{\prime}\right)^{\prime}+$ $a q z=0$ is also oscillatory for functions satisfying $a(t) \geqslant 1$ and

$$
2 p(t) a^{\prime}(t)-3 \int_{0}^{t} p(s) a^{\prime 2}(s) a^{-1}(s) d s
$$

is nonincreasing.
\end{abstract}

In [2] Fink and St. Mary showed that if the second order linear equation

$$
\left(p(t) y^{\prime}(t)\right)^{\prime}+q(t) y(t)=0, \quad t \in[0, \infty),
$$

where $p$ and $q$ are piecewise continuous functions and $p(t)>0$, is oscillatory then the following equation

$$
\left(p(t) z^{\prime}(t)\right)^{\prime}+\lambda q(t) z(t)=0, \quad t \in[0, \infty),
$$

is also oscillatory for any $\lambda>1$. This in fact follows immediately from the classical Picone-Sturm Comparison Theorem if we observe that (2) is equivalent to

$$
\left(\frac{p(t)}{\lambda} z^{\prime}(t)\right)^{\prime}+q(t) z(t)=0
$$

the leading coefficient of which is smaller than that of (1).

Erbe in [1] then observed that multiplying the coefficient $q$ by a certain class of (nonconstant) functions $a(t)$ preserves the oscillatory property, thus extending the result of Fink and St. Mary. The condition on $a$ is that $\left(p(t) a^{\prime}(t)\right)$ be nonincreasing and $a(t) \geqslant 1$.

The purpose of this paper is to show that Erbe's result is still true for a wider class of functions $a$, namely those that satisfy

(3) $a(t) \geqslant 1,2 p(t) a^{\prime}(t)-3 \int_{0}^{t} \frac{p(s) a^{2}(s) d s}{a(s)}$ is nonincreasing for large $t$.

This obviously includes Erbe's class of functions since the second condition in (3) is trivially satisfied by such functions.

Received by the editors May 13, 1981.

AMS (MOS) subject classifications (1970). Primary 34C10.

Key words and phrases. Oscillation, second order, linear differential equations.

1 The author wishes to thank the Graduate School of Northern Illinois University for a grant that partially supports this research. 
LEMMA 1. Let $f$ be a positive nonincreasing function and $g$ a positive $C^{1}$ function, then the function

$$
f(t) g(t)-\int_{0}^{t} f(s) g^{\prime}(s) d s
$$

is nonincreasing.

Proof. If $f$ is $C^{1}$, the lemma is obvious since

$$
f(t) g(t)-\int_{0}^{t} f(s) g^{\prime}(s) d s=\int_{0}^{t} f^{\prime}(s) g(s) d s+f(0) g(0) .
$$

If $f$ is not $C^{1}$, we can approximate it by a smooth nonincreasing function and prove the lemma by a continuity argument.

LEMMA 2. Suppose that a(t) satisfies the second condition of (3), then the function

$$
\left(\frac{p(t) a^{\prime}(t)}{2 a^{2}(t)}+\int_{0}^{t} \frac{p(s) a^{\prime 2}(s) d s}{4 a^{3}(s)}\right)
$$

is a nonincreasing function of $t$.

Proof. This follows from Lemma 1 by letting

$$
f(t)=2 p(t) a^{\prime}(t)-3 \int_{0}^{t} \frac{p(s) a^{\prime 2}(s) d s}{a(s)}
$$

and $g(t)=1 / 4 a^{2}(t)$.

THEOREM 1. Let a be a $C^{1}$ function on $[0, \infty)$ satisfying (3). If equation (1) is oscillatory, then the following equation

$$
\left(p(t) z^{\prime}(t)\right)^{\prime}+a(t) q(t) z(t)=0
$$

is also oscillatory.

REMARKs. 1. Erbe's Theorem was formulated with a different leading coefficient $p_{1}(t)$ in (4) such that $p_{1}(t) \leqslant p(t)$. Since this slightly more general form follows easily upon applying the Picone-Sturm Comparison Theorem to the above form we prefer to state our result in the simplified form.

2. From the proof it is obvious that in fact a Comparison Theorem is valid on finite intervals, i.e., in between any two zeros of any solution $y$ of (1) there is at least one zero of any solution of (4).

3. If the function $a$ is $C^{2}$, then the second condition in (3) is equivalent to

$$
3 p(t) a^{\prime 2}(t) \geqslant 2\left(p(t) a^{\prime}(t)\right)^{\prime} a(t) \text { for large } t .
$$

Proof. Let $r(t)=-p(t) z^{\prime}(t) / z(t)$. It satisfies the Riccati equation

$$
r^{\prime}(t)=a(t) q(t)+r^{2}(t) / p(t) .
$$

The substitution $R(t)=r(t) / a(t)$ transforms this to

$$
R^{\prime}(t)=q(t)+\frac{a(t)}{p(t)}\left(R^{2}(t)-\frac{p(t) a^{\prime}(t) R(t)}{a^{2}(t)}\right) .
$$


If equation (4) has a solution with no zeros in $[c, \infty)$, then the corresponding function $R$ satisfies the Riccati integral equation

$$
R(t)=R(c)+\int_{c}^{t} q(s) d s+\int_{c}^{t} \frac{a(s)}{p(s)}\left(R^{2}(s)-\frac{p(s) a^{\prime}(s) R(s)}{a^{2}(s)}\right) d s
$$

on $[c, \infty)$. Finally the substitution $\rho(t)=R(t)-p(t) a^{\prime}(t) / 2 a^{2}(t)$ results in the Riccati equation

$$
\begin{aligned}
\rho(t) & =\alpha+\int_{c}^{t} q(s) d s-\frac{p(t) a^{\prime}(t)}{2 a^{2}(t)}-\int_{0}^{t} \frac{p(s) a^{\prime 2}(s) d s}{4 a^{3}(s)}+\int_{c}^{t} \frac{a(s)}{p(s)} \rho^{2}(s) d s \\
& =\alpha+\int_{c}^{t} q(s) d s+Q_{1}(t)+\int_{c}^{t} \frac{a(s)}{p(s)} \rho^{2}(s) d s
\end{aligned}
$$

where $Q_{1}$ is a nondecreasing function, by Lemma 2 . We may take $Q_{1}$ to be $C^{1}$ for otherwise we can approximate it by a $C^{1}$ function and use a continuity argument. Equation (6) corresponds to the linear equation

$$
\left(\frac{p(t)}{a(t)} Z^{\prime}(t)\right)^{\prime}+\left[q(t)+q_{1}(t)\right] Z(t)=0
$$

where $q_{1}(t)=Q_{1}^{\prime}(t) \geqslant 0$. Since (6) has a solution on $[c, \infty)$, (7) is nonoscillatory. The Picone-Sturm Comparison Theorem shows that, since $p(t) / a(t) \leqslant p(t)$ and $q(t)+q_{1}(t) \geqslant q(t)$, (1) is nonoscillatory, contradicting our hypotheses. This completes the proof.

A repeated application of Theorem 1 gives a more general result.

THEOREM 2. Let $a_{1}, a_{2}, \ldots, a_{n}$ be functions satisfying condition (3) and suppose that (1) is oscillatory, then

$$
\left(p(t) z^{\prime}(t)\right)^{\prime}+\left(\prod_{i=1}^{n} a_{i}(t)\right) q(t) z(t)=0
$$

is oscillatory.

An example of a function $a(t)$ satisfying (3) with $p(t)=1$ but not Erbe's condition is $a(t)=\alpha t^{1 / 2}+t^{-3 / 2} \sin t$, for any $\alpha \in(1 / 2,1)$.

Applying Theorem 1 to any known oscillation criterion, we can obtain new oscillation criteria, for example,

COROLlARY 3. Let a be any function satisfying condition (3) and suppose the function $Q_{a}(t)=\int_{0}^{t} q(s) / a(s) d s$ satisfies the condition:

for any $\lambda>0$, the set $\left\{t \in[0, \infty)=Q_{a}(t) \geqslant \lambda\right\}$ has infinite measure,

then the equation $z^{\prime \prime}(t)+q(t) z(t)=0$ is oscillatory.

In particular $\lim _{t \rightarrow \infty} \int_{0}^{t} q(s) / a(s) d s=\infty$ is sufficient for oscillation.

COROLlary 4. Let a satisfy (3), then

$$
\lim _{t \rightarrow \infty} \int_{0}^{t} \frac{s^{\alpha} q(s)}{a(s)} d s=\infty
$$

for some $\alpha \in(0,1)$ implies that $z^{\prime \prime}(t)+q(t) z(t)=0$ is oscillatory. 
Proof. We compare the given equation with the equation $y^{\prime \prime}(t)+q(t) y(t) / a(t)$ $=0$. That the hypotheses of the two corollaries are sufficient respectively to guarantee that the latter equation is oscillatory is well known, see for example [3].

\section{REFERENCES}

1. L. Erbe, Oscillation theorems for second order linear differential equations, Pacific J. Math. 35 (1970), 337-343.

2. A. M. Fink and D. F. St. Mary, A generalized Sturm comparison theorem and oscillation coefficients, Monatsh. Math. 73 (1969), 207-212.

3. M. K. Kwong and A. Zettl, Integral inequalities and second order linear oscillation, J. Differential Equations (to appear).

Department of Mathematics, Northern Illinois University, DeKalb, Illinois 60115 